\title{
Anemia Autoinmune y Trombopenia en Enfermedad Mixta del Tejido Conectivo.
}

\author{
Drs. Eduardo Takesnik G. ${ }^{\text {, }}$, Hernán Taboada L. ${ }^{1}$, Santiago Rivero D. ${ }^{2}$ y Sergio Jacobelti Gr ${ }^{2}$
}

Autoimmune anemia and thrombocytopenia in Mixed Connective Tissue Disease.

\begin{abstract}
A six years old boy with Mixed Connective Tissue Disease is presented. Initial manifestations were Coombs positive hemoly tic anemia, thrombocy topenia, Arthritis, liver and spleen entargment.

Search for antibodies to extractable nuclear antigens (ENA) was positive for the ribonuclease senisitive component (anti-RNP).

This syndrome improved under corticosteroid therapy.

To our knowledge this is the first report of sucl onset of MCTD in children.

Clinical symptoms, laboratory manifestations and treatment of MCTD in children are analized.
\end{abstract}

La asociación de anemia hemolítica y trombocitopenia es infrecuente en pediatría. Evans la describió en $1951^{3}$ y puede ser primaria ${ }^{2}$ o secundaria a cirrosis hepática, leucemia, tiroiditis de Hashimoto ${ }^{2}$, etc. En patologia reumatológica se ha descrito en lupus eritematoso diseminado $3-4-5$, esclerodermia ${ }^{6}$ y existen dos referencias bibliográficas en pacientes adultos con enfermedad mixta del tejido conectivo (EMTC) ${ }^{7-a}$.

En 1972 Sharp? describió la enfermedad mixta del tejido conectivo como un síndrome con caracteristicas clínicas combindas de lupus eritematoso diseminado, esclerodermia y polimiositis y que tiene, en el suero, títulos altos de anticuerpos contra un antígeno soluble (ENA), especificamente una ribonucleoproteina (RNP) de origen nuclear. Hasta ahora se han descrito aproximadamente cincuenta casos en pacientes pediátricos $9 \cdot 10$. $11-12-13 \cdot 14 \cdot 15-16-17$.

El objetivo de este trabajo es informar del caso clínico de un paciente pediátrico portador de anemia hemolítica y trombopenia secundaria a una Enfermedad Mixta del Tejido Conectivo (E.M.T.C.).

\section{CASO Clinico}

C.U.M. Idad: 6 años; varón

Consultó por primera vez a los 3 anos 9 meses por fiebre de diez dias de duración, falta de ánimo,

1 Departamento de Pediatría Lniversidad Católica de Chile. Servicio de Pediatría Hospital Sótero del Río.

2 Departamento de Enfermedades Endocrinológicas, Metabólicas y Reumatológicas, Escuela de Medicina, Lniversidad Católica de Chile. anorexia y dolor abdominal difuso. En el examen físico habia hepato y esplenomegalia. Las anamnesis remota y familiar fueron negativas. Evolucionó con temperaturas febriles, de hasta $38^{\circ} \mathrm{C}$, diurnas, durante una semana, posteriormente se hizo afebril y permaneció sin sintomas hasta aproximadamente los 4 años 3 meses, cuando presentó, de modo progresivo, astenia, anorexia, dolor abdominal difuso y artralgias de rodillas e interfalángicas. Se encontró el hígado $4 \mathrm{cms}$ bajo el reborde costal (proyección hepática de $12 \mathrm{cms}$ ), bazo $3 \mathrm{cms}$ bajo el reborde costal $y$ consistencia aumentada y artritis fugaz de rodilia izquierda. Se documentó por primera vez pancitopenia. Durante los tres siguientes meses aumentaron su astenja, adinamia y anorexia, se agregó un sindrome de anemia rápidamente progresiva $y$ aparecieron petequias y equimosis. Estuvo nuevamente febril y con artralgias difusas.

En su primera consulta tenía VHS: $79 \mathrm{~mm} / \mathrm{ho-}$ ra, hemograma, sedimento de orina, bilirubina, fosfatasas alcalinas, transaminasas, tiempo de protrombina, Radiografía de tórax, Urocultivo, coprocultivo, hemocultivos y reacción de aglutinación para salmonella typhi, y brucella nomales.

A los 4 años 6 meses el estudio de laboratorio mostró hematocrito(hto); $27 \%$, reticulocitos; $7 \%$, anisocitosis leve, normocromía, glóbulos blancos $3.400 \mathrm{~mm}^{3}$, plaquetas $25.000 \mathrm{~mm}^{3}$, Coombs directo positivo, Fierro sérico $36 \mathrm{~kg} / \mathrm{dl}$., TIBC $262 \mu \mathrm{g} / \mathrm{dl}$., saturación de transferrina $13,7 \%$; mielograma: hiperplasia eritroide relativa. Resultaron negativas las reacciones de hemaghtinación para enfermedad de Chagas, hemaglutinación y fijación del complemento pard toxoplasmosis y antígeno australiano. No se encontró sangre oculta en deposiciones (examen de Guayaco). Se obtuvo valores 
normales de hemoglobinemia, resistencia osmótica de los glóbulos rojos, electroforesis de hemoglobina y prueba de estabilidad de hemoglobina. La radiografia de huesos largos no mostró alteraciones.

Los exámenes inmunológicos (Tabla 1) realizados demostraron hipergamaglobulinemia con IgG elevada en la determinación de inmunoglobulinas séricas. El factor reumatoídeo y las células de lupus resultaron negativos. $\mathrm{C}_{3}$ estaba dentro de límites normales, sin embargo los anticuerpos antinucleares (AAN) y los anticuerpos anti ENA (Ac anti ENA) estos últimos específicos para ribonucleoproteina (RNP), detectados por hemaglutinación pasiva y tratamiento enzimático del ant ígeno ENA fueron positivos.

Antes de comenzar el tratamiento del paciente, una nueva prueba de Coombs directa fue positiva $y$ un hemograma reveló un hto. de $12 \%$, reticulocitos $10 \%$, leucocitos $7.300 \mathrm{~mm}^{3}$ y ausencia de plaquetas en el frotis.

El tratamiento realizado se resume en la Figura 1. lnicialmente se usó prednisona en dosis de $6 \mathrm{mgr} \mathrm{kg}$ día, fraccionada en tres dosis diarias, en coincidencia se observó rápida respuesta, consistente en disminución de visceromegalia, anemia y púrpura y normalización del hemograma, lo que indujo a reducir las dosis de prednisona progresivamente. Desde el cuarto mes de tratamiento recibe esteroides en días alternos excepto por algunos djas (debido a una infección intercurrente) al promediar el sexto mes de terapia, en que se usaron diariamente. El tratamiento actual es de

Tabla 1.

Anemia Autoinnune y Trombopenia en E.M.T.C. Exámenes inmunológicos

$$
C_{3} A A N \text { Acanti DNA Ac anti ENA }
$$

\begin{tabular}{|c|c|c|c|c|}
\hline $\begin{array}{l}4 / X 11 i 80 \\
(4 \text { a } 8 \mathrm{~m})\end{array}$ & 180 & $(+) 1 / 200 \mathrm{M}$ & $2 \%$ & () \\
\hline $\begin{array}{l}12 / 1 / 81 \\
(4 \mathrm{a} 9 \mathrm{~m})\end{array}$ & 100 & $(+) \leqslant / 200 M$ & $5 \%$ & $\mathrm{RNP}: / 800$ \\
\hline $\begin{array}{l}26 / 1 / 81 \\
\left(4 \mathrm{a}^{\mathrm{m}}\right)\end{array}$ & & $(+) 1 / 100 \mathrm{M}$ & $(-)$ & RNP $1 / 2048$ \\
\hline $\begin{array}{l}20 / \mathrm{N} / 8 \mathrm{I} \\
(5 \mathrm{a} \mathrm{Im})\end{array}$ & & $(-)$ & $4 \%$ & $\operatorname{RNP} 1 / 256$ \\
\hline $\begin{array}{l}27 / \times 1 / 81 \\
(5 \text { a } 7 \mathrm{~m})\end{array}$ & 164 & (+) $\mathrm{t} / 400 \mathrm{M}$ & $7 *$ & $(-)$ \\
\hline $\begin{array}{l}9 / \mathrm{VII} / 82 \\
(6 \text { a } 2 \mathrm{~m})\end{array}$ & & (+) $1 / 100$ & $8 \%$ & $\begin{array}{l}\text { KNP } 1 ; 400 \\
\text { So - }\end{array}$ \\
\hline
\end{tabular}

$\mathbf{s}=$ patrón de inmunofluorescencial moteado

Sn: Anticuerpos dirigidos contra una glicopjoteina llamada Sm

AAN: Anticuerpos antinucleares

DNA: Acido desoxiribunucleico

ENA: Antígeno solublc

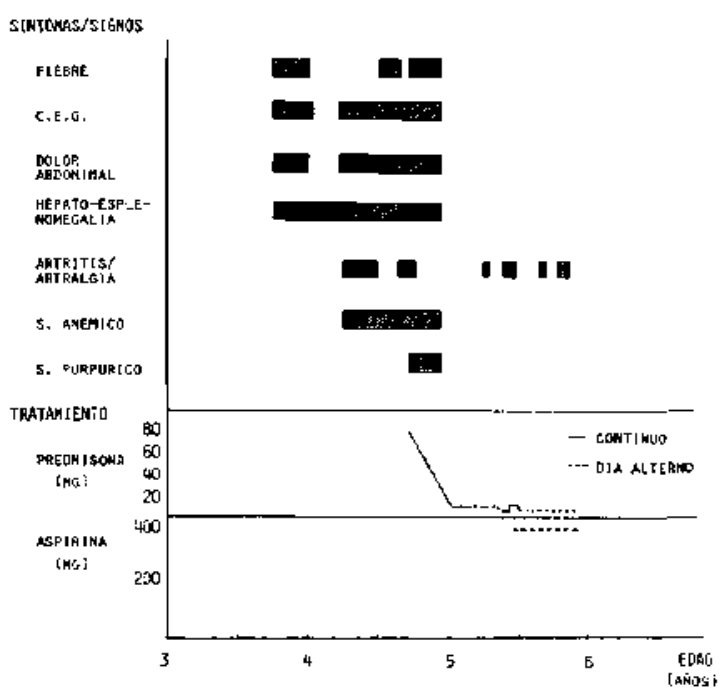

Figura 1.

Aremia autoinmune y trombopenia en E.M.T.C. Expresión gráfica de síntomas y signos clínicos, evolución y tratamiento.

prednisona 5 mgrs. día por medio y aspirina $400 \mathrm{mgr}$. dia por medio.

Su evolución ha sido satisfactoria, ha permanecido en buenas condiciones generales, con artralgias ocasionales, fugaces, de hombros, muñecas y tobillos. Su ritmo de crecimiento es adecuado desde que ingiere esteroides en dias alternos y el examen físico es normal.

Los hemogramas de control, el sedimento de orina, depuraciớn de creatinina y los estudios relacionados con la integridad hepática, muscular y cardíaca han sido normales.

La evolución de los exámenes inmunológicos se aprecia en la Tabla 1.

La reacción de Coombs directa ha sido permanentemente positiva.

\section{DISCUSION}

En primer término cabe destacar las dificultades para hacer el diagnóstico en este paciente por lo poco específico de sus síntomas y signos iniciales, pues sólo después de aproximadamente un afto de evolución, apareció el compromiso articular el que tampoco fue nuy llamativo. La asociación de este último con anemia hemolítica y trombopenia permitió sospechar una mesenquimopatía. La literatura señala que en pacientes pediátricos la E.M.T.C. se presenta con una gran variabilidad clínica ${ }^{15}$, la aparición de síntomas y signos es lenta y Singren ${ }^{9}$. calculó un promedio de cuatro años entre las primeras manifestaciones y el diagnóstico correcto. 
Se catalogó a este paciente de E.M.T.C. por las manifestaciones clínicas y anticuerpos anti RNP en titulos significativos: estos se presentan en forma constante en la E.M.T.C. $y$ constituyen un elemento fundamental en el reconocimiento de esta afección ${ }^{18-19}$. La buena evolución clínica de nuestro paciente es otro apoyo al diagnóstico planteado. Los anticuerpos anti RNP no son específicos de esta afección y suelen aparecer en alrededor del $25 \%$ de pacientes portadores de lupus eritematoso generalizado confirmado, aunque en estos casos los títulos son menores y su presencia sugiere mejor pronóstico, especialmente por menor frecuencia e intensidad del compromiso renal ${ }^{19}$; el papel protector del Anticuerpo anti RNP en el desarrollo de la nefropatía lúpica no ha sido actarado en sus mecanismos patogéricos 20 .

La noción de E.M.T.C. como una entidad clínica diferente del resto de las enfermedades difusas del tejido conectivo ha sido discutida, aunque la opinión actual más generalizada tiende a considerarla como una enfermedad definida y distinta del resto.

En pacientes pediátricos la mortalidad calculada a 5 años de la E.M.T.C. es $20 \%$, menor que la del lupus eritematoso diseminado que alcanza hasta $64 \% 15$.

Las caracteristicas clínicas de E.M.T.C. en niños y aduitos, se muestran en la Tabla 2. Lo más frecuente es poliartritis y fenómeno de Raynaud. La miositis ocurre más en nî̉os que en adultos ${ }^{15}$.

Tabla 2.

Aremia Autoimmune y Trombopenia en E.M.T.C. Caracteristicas clínicas en pacientes adultos y pediatricos.

Edad (años)

$$
\begin{array}{ll}
\text { Niños } & \text { Adultos } \\
\text { No }=50 & \text { No }=184
\end{array}
$$

Promedio (rango)

$$
12.8(4-21) \quad 32.1(22-80)
$$

\section{PORCENTAJE}

Sexo femenino

Artritis/artralgia

86

92

Fenómeno Raynasd

Enf. Pulmonar intersticial

78

40

Mjositis

Cambios cutaneos tipo esclerodermia

Cardiopatia

Anormalidades motilidad esófagica

Nefropatía

\section{4}

30

34

28
86

89

89

38

56

32

10
La presentación bajo la forma de anemia hemolítica severa, trombopenia y reacción de Coombs Positiva a diferencia con lo comunicado en adultos, no fue encontrada por los autores en la literatura pediátrica.
En pacientes adultos portadores de lupus eritematoso generalizado, la presencia de anemia hemolítica $y / 0$ trombopenia se ha asociado con mejor pronóstico y menos incidencia de nefropatia, alteraciones neuro psiquiátricas e hipo-complementemia persistente ${ }^{3}$. La trombopenia y la anemia son de tipo inmune, ya que se han detectado anticuerpos antiplaquetarios y antieritrocíti$\cos$, estos últimos pertenecientes a las distintas clases de inmunoglobulinas, pero en los casos de anemia hemolitica clinica son principalmente de tipo IgG y capaces de fijar complemento $21 \cdot 22$

La respuesta al tratamiento con estetoides, tal como en nuestro caso, se describe como favorable $^{7-15}$, en especial en aquellos pacientes en que predominan las manifestaciones de miositis, artritis, serositis, linfodenopatia, hepatoesplenomegalia, fiebre, anemia y leucopenia ${ }^{7}$. En el caso de un adulto, semejante a nuestro paciente, se describió también buena respuesta a los esteroides ${ }^{8}$.

\section{RESUMEN}

Se presenta un varón de seis años, portador de enfermedad mixta del tejido conectivo (EMTC). Las manifestaciones iniciales fueron: anemia hemolítica Coomb positivo, trombopenia, artritis $y$ hepatoesplenomegalia.

La búsqueda de anticuerpos dirigidos contra un antígeno soluble (ENA) dio resultados positivos contra una ribonucleoproteína (RNP) de origen nuclear.

El paciente mejoró en coincidencia con e] empleo de prednisona.

No encontramos en la literatura otra comunicación de EMTC con esta forma de comienzo en niños.

Se revisa las manifestaciones clínicas, exámenes de laboratorio y tratamiento de la E.M.T.C. en niños.

\section{REFERENCIAS}

I Evans R.S., Tokahashi K., Dunne R.T., Payne R., lie $C . K$. Primary Thrombocy topenia purpura and acquired hemolytic aremia. Arch Int. Med. 87: 48, 1951.

${ }^{2}$ Chang-Hon pui, Willians H., Wang W. Evank syndrome in childhood J. Pediatr. 97: 754, 1980.

3 Alger $M$, Alarcón-Segovia D., Rivero $S$. Hemolytic anemia and thrombocy topenic purpura: two related subsets of Systemic Lupus Erythematosus. J. Rheum. 4: $351,1977$.

4 Favre H. Chatelanot F., Miescher P. Autoirmunc hematological disease associated with infraclinical Systemic Lupus Erythematosus in four patients. Arr. J. Med. 66: 91, 1979 .

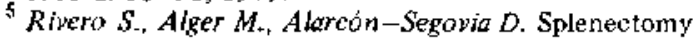
for hemocy topenid in Systemic Lupus Erythematosus. Arch. Intern. Med. 139: 773, 1979. 
${ }^{6}$ Ivey KJ., Hwang Y.F., Sheets R.F. Scleroderma associated with thrombopenia and Coombs (t) hemolitic anemia. Am. J. Med. 51: 815, 1971.

${ }^{7}$ Sharp G.C., Irving W.S., Tan E.M., et ol. Mixed connective tissue disease; and apparently distinct theumatic disease syndrome associated with a specific antibody to an extractable nuclear antigen (ENA). Am. J. Med. s2: $148,1972$.

${ }^{6}$ Segond P., Yeni P., Jacquot J., Massias P. Severe attoimmune anemia and thrombopenia in mixed connective tissue disease. Arthritis Rheum 21: 995, 1978.

9 Singsen B., Konreich H., Koster-King K., Brink S., Bernstein B., Hanson V., Tan E. Mixed connective tissue disease in children. Arth and Rheum 20. 355, Suppl March 1977.

10 Sanders D.Y., Huntley C.C., Sharp G.C.: Mixed connective tis sue disease in Child. J. Pediatr. 83:642, 1973.

11 Fraga A., Gudino J., Ramos Niembro F. Mixed connective tissue disease in childhood. Am. J. Dis. Child. 132: 263, 1978 .

12 Singsen B.H., Bernstein B.H., Kornreich H.K. et al: Mixed connective tissue disease in childhood. J. Pediatr. 90: 893, 1977.
${ }^{3}$ O'Connel D.J., Bennet R.M.: Mixed connective tissue disesse, clinical and radjological aspects of 20 cases. Br. J. Radiol. 150: 620, 1977.

14 Detgen W.J., Boice J., Lowless $O$. Mixed connective tissue disease in children and adolescents. Pediatrics 67: 3331981 .

15 Singsen B.H., Swanson V., Bernstein B.H., Heuser E., Hanson $V$, Landing $B$. A histologic evaluation of Mixed connective tissue disease in childhood. Am. J. Med. $68: 710,1980$.

16 Michels H., Schuchmann L. Das Sharp syndrom (MCTD) eine besondere form der kollagenose in kindesalter. Klin Paediat 192: 389, 1980.

17 Notman D.D. Kurata N. Tan E. Profiles of antituclear antibodies in systemic rheumatic disease. Ann. Inter. Med. 83: 464, 1975.

18 Rivero $S$., Jacobelli $S$. Rev. Med. Chile 110: 433 , 1982.

${ }^{19}$ Reichlin $M$. Problems in diferentiating SLE and mixed connective tissue disease. N. Engl. J. Med. 295: 1194 1976.

20 Schreiber $A$. Autoimmune hemolytic anemia. Ped. Cin. N.A. $27: 253,1980$.

21 Stiter D.D., Stabo J.D., Fudenberg H.H., Weth J.V. Basic and clínical lmmunology, Lange 1982. 